\title{
Effects of Neoadjuvant Chemotherapy in High-Grade Non-Metastatic Osteosarcoma of Extremities
}

\author{
Milan Samardziski ${ }^{1}$, Vesna Janevska ${ }^{2}$, Beti Zafirova-Ivanovska ${ }^{3}$, \\ Violeta Vasilevska ${ }^{4}$ and Slavica Kraleva ${ }^{5}$ \\ ${ }^{1}$ University Clinic for Orthopaedic Surgery, Skopje \\ 2Institute for Pathology, Skopje \\ 3Institute for Epidemiology and Biostatistics, Skopje \\ ${ }^{4}$ University Surgical Clinic "St. Naum Ohridski", Skopje \\ Institute for Radiology and Oncology, Skopje \\ Macedonia
}

\section{Introduction}

Osteosarcoma is a very rare malignant bone tumor with an incidence of 4-6 cases in $1,000,000$ inhabitants and appears mostly in the young and active population aged 10- 30 years (Price \& Jeffree, 1977). Amputations and disarticulations as dominant treatment for malignant bone tumors in the beginning of $20^{\text {th }}$ century are rarely and very selectively used today. Before 1970, amputation was the primary treatment for high-grade osteosarcoma and $80 \%$ of patients died of lung metastatic disease. Despite aggressive and radical surgery, 5year survival was low (10-20\%) (Rosen et al., 1976). Introducing new sophisticated diagnostic methods (CT and MRI) gave the possibility of precise anatomic definition of the tumors and the borders of infiltration into the surrounding tissues. Better planning of the biopsy and the definite operative procedure, and fostering better patient selection for specific treatment strategies, can decrease the risk of tumor spread into the surrounding tissue and lower the risk of distant metastases. After 1980, improvement of chemotherapeutic protocols with neoadjuvant chemotherapy, better preoperative planning and modern reconstructive options after resection of osteosarcoma led to better survival rates of patients with limb-sparing procedures (Bacci et al., 1993, Bruland \& Phil, 1999).

Currently, $80-85 \%$ of the patients with osteosarcoma on the extremities can be safely treated with wide resection and limb preservation (Di Caprio et al., 2003). A multidisciplinary approach to diagnosis and treatment, combination chemotherapy and a number of options for reconstruction after osteosarcoma resection (especially in chemotherapy-sensitive tumors) have increased long-term survival rates from 60 to $80 \%$. Amputations, once a dominant treatment for malignant bone tumors, now are rarely and very selectively used. Most patients with extremity-localized osteosarcoma are candidates for limb-sparing procedures because of the: effective chemotherapeutic agents and regimens, the improved imaging modalities, and advances in reconstructive surgery. 
Application of neoadjuvant chemotherapy improves survival rates and functional outcome in patients with non-metastatic, high-grade osteosarcoma of the extremities (Wittig et al., 2002).

Before consideration of limb preservation, the patient needs to be appropriately staged and assessed through a multidisciplinary approach. Some elements of the disease may warrant concern, including relative contraindications to such procedures. However, surgical treatment associated with a limb-sparing operation is also associated with a significant number of complications and requires extensive rehabilitation. The main risk of limbsalvage procedures is that complications sometimes may cause a delay of chemotherapy (Sæter al., 1996).

\section{Osteosarcoma subtypes and characteristics}

Depending on cytological or histo-pathological features of the tumor matrix or tumor cells, osteosarcomas are divided into two groups. In the first group there are patients with lowgrade osteosarcoma and surgery alone has the primary role of treatment. In the second group there are patients with high-grade osteosarcoma. In this group of patients "sandwich therapy" is strongly preferred (neoadjuvant chemotherapy - surgery - adjuvant chemotherapy) (Enneking, 1975; Bacci et al., 1993; Messerschmitt et al., 2009).

\subsection{Intramedullary osteosarcoma}

Conventional or "classic" osteosarcoma is the most prevalent type in children and adolescents (up to $80 \%$ of all cases). This type of osteosarcoma originates from the intramedullary cavity and is typically high-grade (Fig. 1a). An osteoblastic and/or osteolytic lesion with vast cortical destruction and various amount of soft tissue extension dominates on X-rays. Histopathologic examination demonstrates malignant mesenhimal cells, spindle to polyhedral in shape, with pleomorphyc nuclei and occasional mitotic figures. Evidence of direct bone or osteoid production from the mesenhim is crucial for diagnosis (Fig. 1b, 1c). World Health Organization has further subcategorized high-grade intramedullary osteosarcoma since 2002, depending on the predominant extra cellular matrix on: osteoblastic (approximately $50 \%$ of cases), chondroblastic ( $25 \%$ of cases) or fibroblastic (25\% of cases). (Fletcher et al., 2002).

Teleangiectatic osteosarcoma is a rare variant accounting for approximately $4 \%$ of all osteosarcoma cases in children and adolescents. Very often they are associated with pathological fracture of the first presentation. Eccentric osteolytic lesion on the metaphysis, with destruction and expansion of the eroded cortex dominates on x-ray (Fig. 1b). Histopathologic examination reveals a malignant tumor with multiple dilated hemorrhagic sinuses as well as a scarce amount of high-grade osteosarcoma cells and rare osteoid formation within the septa. These radiographic and histo-pathologic features resemble an aneurismal bone cyst which is cdaracteristic.

Low-grade intramedullary osteosarcoma constitutes 1 to $2 \%$ of all osteosarcoma cases and generally affects patients in the third or fourth decade. Lesions most commonly affect the distal femur and proximal tibia, with relatively unaggressive radiographic appearance, resembling fibrous dysplasia ("fibrous dysplasia-like" osteosarcoma). Histo-pathological features consist of well-differentiated cells dispersed within woven microtrabeculae of bone and fibrous stroma. Small amounts of osteoid, mitotic atypia and mitoses can also be seen (Fletcher et al., 2002). 
Small-cell osteosarcoma is a rare variant constituting $<1.5 \%$ of all osteosarcoma cases. This subtype is similar to the high-grade osteosarcoma, with the same site or age distribution and aggressive biologic behavior. The lesion is osteolytic with destruction of cortex and variable sclerosis. MRI reveals large spindle or circumferential tumor mass, similar to Ewing sarcoma. Small, round, malignant cells within an osteoid matrix make the histo-pathological diagnosis problematic. To differentiate this osteosarcoma from Ewing sarcoma, direct mesenhimal production of osteoid must be found, because this osteosarcoma is positive for CD 99 immuno-histochemical stains (Fletcher et al., 2002).

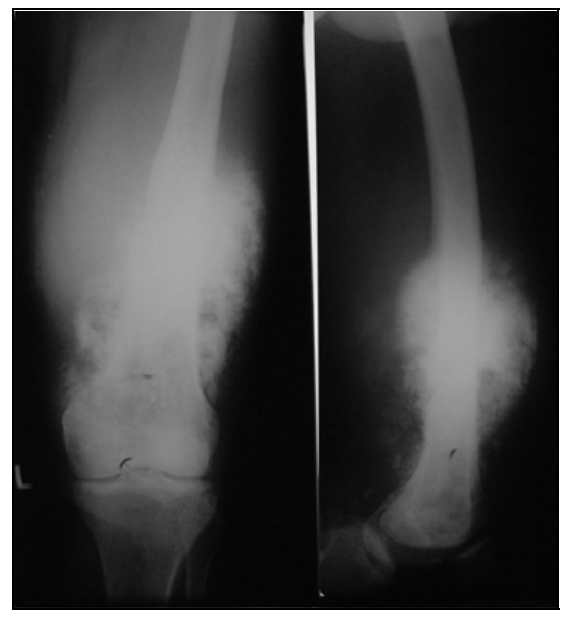

a)

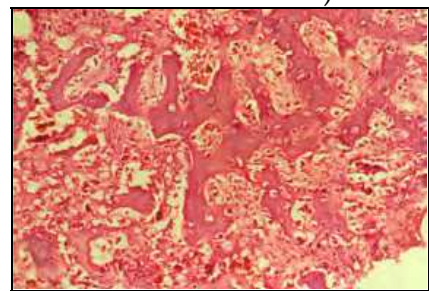

c)

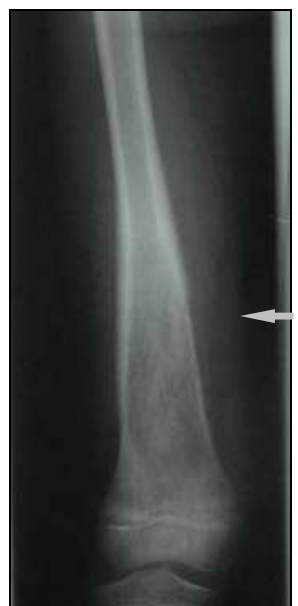

b)

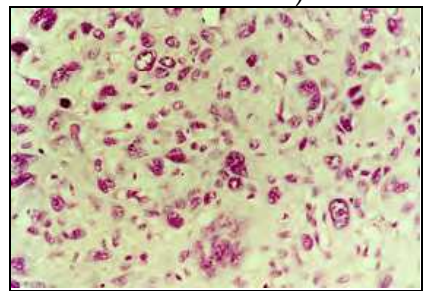

d)

Fig. 1. a) X-ray of conventional intramedullary osteosarcoma (osteoblastic lesion with vast cortical destruction and soft tissue edema visible on $\mathrm{x}$-rays); b) X-ray of teleangiectatic osteosarcoma with osteolytic lesion on the metaphysis of distal femur, destruction, expansion of the eroded cortex and Codman's periosteal reaction (arrow); c) Typical histopathological feature of osteosarcoma is osteoid formations directly from the mesenhime; d) Atypical osteoid formation in high-grade anaplastic osteosarcoma typifies the diagnosis.

A few osteosarcomas (less than 1\% of all cases) have so many giant cells that they can be mistaken for giant cell tumors. Cytological atypia of the mononuclear cells can be very subtle and rare. It is important to remember the possibility of a giant cell-rich osteosarcoma when giant cell tumor-like lesion occurs in an unusual location and age, such as the metaphysis in children (Unni, 1998). 


\subsection{Surface osteosarcoma}

Parosteal osteosarcoma arises on the outer surface of the long bone metaphysis, sparing the medullary canal (Fig. 2a). The peak incidence is in the second and third decade, affecting more females than males.(10) Parosteal osteosarcoma is most commonly seen as a juxtracortical variety and constitutes 1 to $6 \%$ of all osteosarcoma cases. Radiographs classically show densely ossified and lobulated mass on the posterior surface of the femur. Sometimes slow-growing tumors may encircle the bone. A low-grade, well differentiated fibrous stroma with osseous components is regularly seen on the histo-pathologic examination. Parallel orientation of trabeculae with additional cartilaginous differentiation is very common (Fig. $2 \mathrm{~d}$ ).

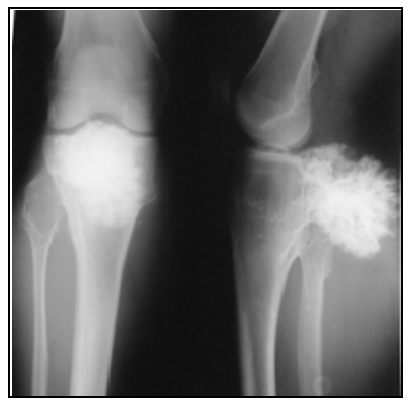

a)

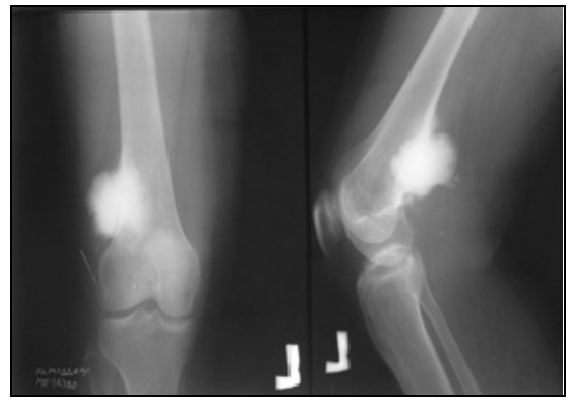

b)

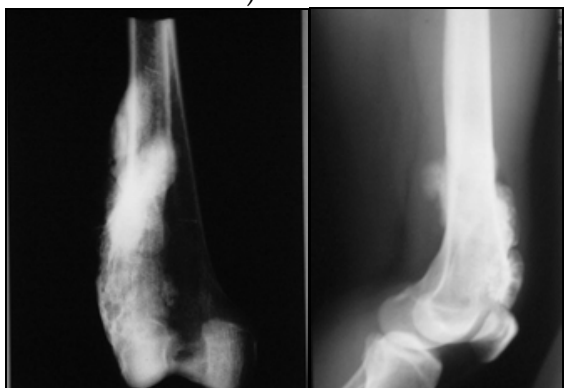

c)

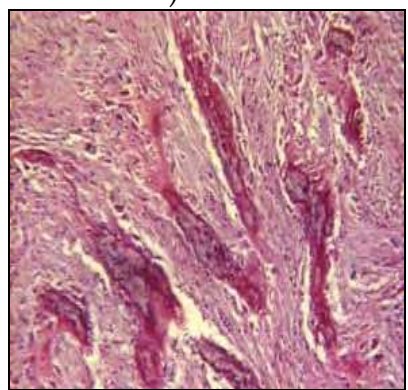

d)

Fig. 2. a) Frontal and lateral x-ray of the periosteal osteosarcoma of right distal femur; b) X-ray in frontal and lateral view of parosteal osteosarcoma of the proximal tibia; c) Frontal and lateral x-ray of high-grade surface osteosarcoma on the right distal femur; d) Parosteal osteosarcoma showing parallel osteoid trabeculae embedded in fibroblastic stroma (HE, x100).

Periosteal osteosarcoma constitutes 1 to $2 \%$ of all osteosarcoma cases and is usually more aggressive than the parosteal variant. A radiolucent lesion is located on the distal femur or proximal tibia, sparing the medullar cavity (Fig. 2b). Codman triangle and "sunburst" periosteal reaction are common radiographic features. Histo-pathologic evaluation demonstrates an intermediate-grade tumor, rich with cartilaginous matrix and rare osteoid fields.

High-grade surface osteosarcoma constitutes $<1 \%$ of all osteosarcomas with the predominant site around the knee. Radiographic analysis shows surface lesion with partial mineralization 
and tumor extension into surrounding soft tissues. In earlier stages of the disease, destruction of the underlying cortex is absent, but with advanced lesions involvement of the medullary cavity is possible (Fig. 2c). The histological features are those of high-grade osteosarcoma, demonstrating spindle cells with atypia and a varying amount of osteoid. A high-grade surface osteosarcoma cannot be differentiated from a conventional osteosarcoma in histological findings alone (Fletcher et al., 2002; Samardziski et al., 2009).

\section{Imaging}

There are various radiological imaging techniques available to achieve an accurate diagnosis and staging of osteosarcoma and to detect local recurrence or distant metastases. Most commonly used are: plain-film radiographs (as "gold" standard), Tc-99m bone scintigrapy, CT of the affected site or of the lungs and CT or conventional angiography. Positron emission tomography (PET-scan) and Thallium scintigraphy have been seldom used due to questionable results in evaluating early osteosarcoma metastases or due to their high-cost (Messerschmitt et al., 2009).

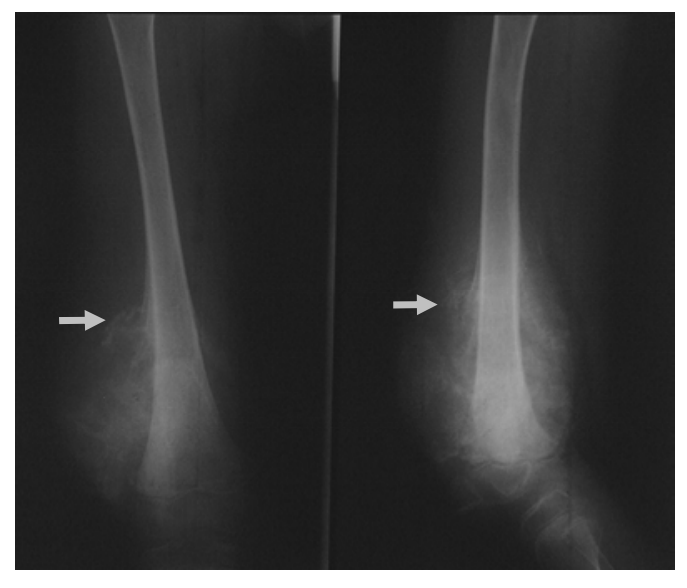

a)

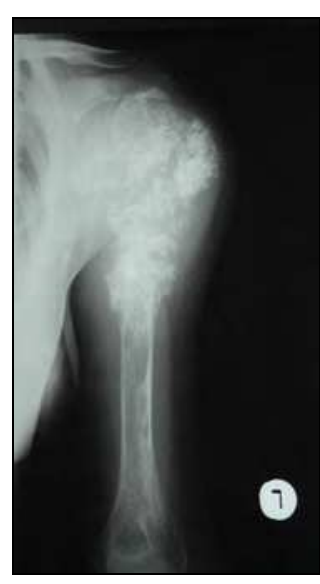

b)

Fig. 3. a) X-ray in two orthogonal planes of typical mixed sclerotic end lytic osteosarcoma of the distal femur. Tumor has penetrated bone and formed a soft tissue mass with Codman's triangles. b) Frontal plane X-ray of osteosarcoma situated on proximal humerus with small, confluent cloud-like densities, destroing the bone completely.

Plain-film radiographs in two ortogonal plains show mixed osteosclerotic and osteolytic tumor, affecting the metaphysis of the bone (although primarily sclerotic or lytic osteosarcomas can occur). The lesion is ill defined from the surrounding bone, affecting and destroing the cortex, with typical small, irregular, confluent, cloud-like densities. If the cortex is completely eroded the lesion forms a soft tissue mass extruding from the bone into the surrounding tissue and may demonstrate ossification detectable on the 
radiographs (Fig. 3). The destruction may be so advanced that pathological fractures or complete bone erosion could be present (Fig. 3b). There is a typical periosteal reaction due to aggressive expansion of the tumor, forming hairy, sun-ray or velvet-like specula of neoplastic bone. In some cases "Codman's triangles" (arrows on Fig. 3a) are present. Plain-film radiographs are used in correlation with bone scintigraphy and CT to detect local recurrence or bone and lung metastases. Additional data for diagnosis and decisionmaking process can be obtained using a "computer assisted diagnosis" in analysis of the x-rays (Lodwick et al., 1963; Samardziski et al., 2004).

Computer tomography (CT) scann of the affected extremity is useful in visualization of the intra and extra-osseous extent of the tumor, especially when extensive necrosis and surrounding edema are present. In this case CT may be superior to MRI. High-definition CT scans can obtain a three dimensional view of the tumor in relation to adjacent neurovascular structures, especially when contrast medium is used (Fig. 4a). All patients with osteosarcoma should undergo CT scanning of the chest and lungs for detection of pulmonary metastases, for diagnosis and staging. After surgery has been performed in patients with non-metastatic osteosarcoma, CT scans of the lung should be repeated every three to six months for two years (Wittig et al., 2002).

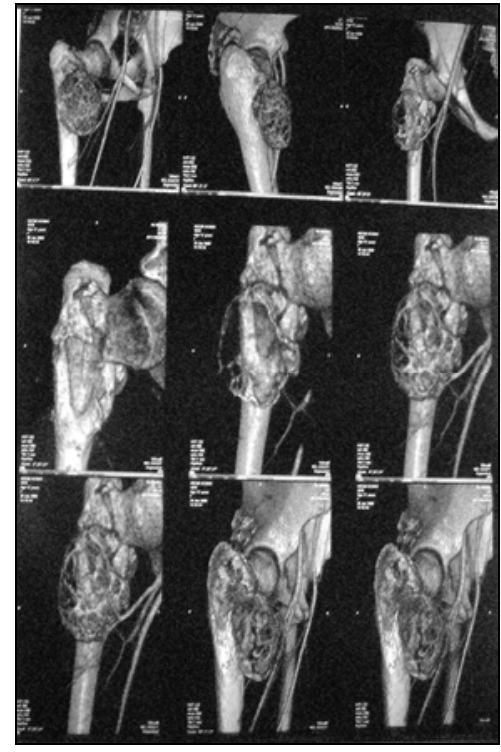

a)

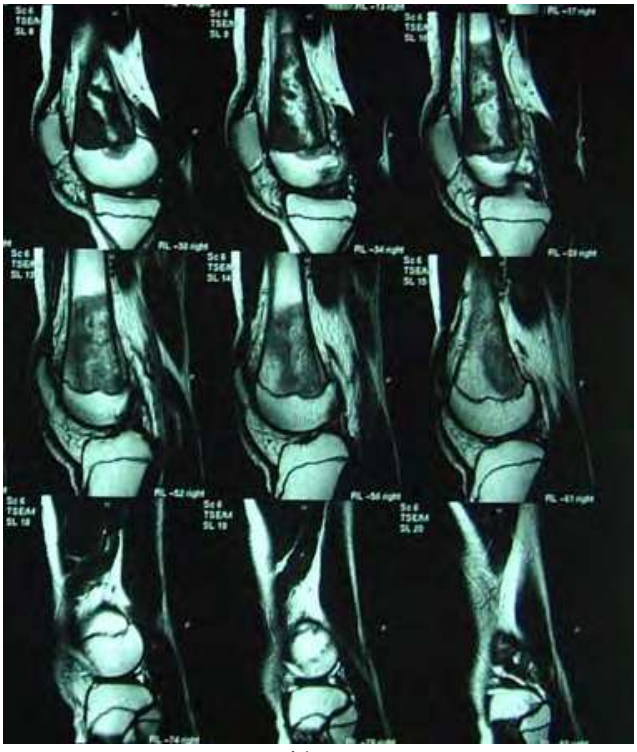

b)

Fig. 4. a) CT scans of proximal femur osteosarcoma, with visualization of the superficial and deep femoral artery b) $\mathrm{T}_{2}$-weighted MRI image of distal femur osteosarcoma with no extraosseous extension.

With magnetic resonance imaging (MRI), standard $\mathrm{T}_{1}$ and $\mathrm{T}_{2}$-weighted and fat-suppressed images are obtained to visualize the affected bone and surrounding tissue with osteosarcoma. Based on MRI studies, the intra-osseous and extra-osseous extent of the 
tumor is visible as well as reactive zone and tissue edema (Fig. 4b). Neurovascular structures and especially neurovascular encasement can be determined and it can help in the process of planning the definite method of treatment. (If encasement is present, amputation or wide resection with vascular reconstruction is obligatory).

Obtaining an MRI prior to surgical resection permits accurate planning of the osteotomy and gross tumor excision (together with the reactive zone) for achieving a "wide" surgical margin. Skip metastases on MRI are easily detectable in the same bone or in the adjacent joint and then a more extensive resection is required. MRI studies are inferior to highdefinition CT scans for lung metastases detection (Di Caprio \& Friedlander, 2003, 2003).

\section{Biopsy and histo-pathological diagnosis}

In spite of the risk for tumor spreading, biopsy is the key step in the diagnosis and treatment of osteosarcoma. Improperly performed biopsy may compromise the treatment plan. It is mandatory to place the biopsy in the line of definite surgical approach for osteosarcoma resection. A specimen taken from the necrotic tissue or from reactive zone (around the osteosarcoma) may be non informative. A large needle biopsy is sometimes preferable, because it is less invasive, with lower risk for skin necrosis, infection and pathological fracture. If no representative osteosarcoma tissue is obtained, an open biopsy will increase the risk of complications or local spreading of the tumor. The best results are achieved when all the biopsy samples are obtained by the same orthopedic oncologist (surgeon) who will perform the definite surgical procedure (Mankin et al., 1982; Campanacci, 1999). One must state that obtaining an accurate histo-pathological diagnosis of the tumor (especially of osteosarcoma) may be very delicate task (Fletcher et al. 2002).

\begin{tabular}{|c|c|c|c|}
\hline Stadium & Grade & Localisation & Metastases \\
\hline IA & \multirow{2}{*}{$\mathrm{G}_{1}$ - Low-grade } & $\mathrm{T}_{1}$ - Intraosseus & $\mathrm{M}_{0}$ - No metastases \\
\hline IB & & $\mathrm{T}_{2}$ - Extraosseus & $\mathrm{M}_{0}$ - No metastases \\
\hline IIA & \multirow[b]{2}{*}{$\mathrm{G}_{2}$ - High-grade } & $\mathrm{T}_{1}$ - Intraosseus & $\mathrm{M}_{0}$ - No metastases \\
\hline IIB & & $\mathrm{T}_{2}$ - Extraosseus & $\mathrm{M}_{0}-$ No metastases \\
\hline IIIA & $\mathrm{G}_{1-2}$ & $\mathrm{~T}_{1}$ - Intraosseus & $\mathrm{M}_{1}$ - With metastases \\
\hline IIIB & $\mathrm{G}_{1-2}$ & $\mathrm{~T}_{2}$ - Extraosseus & $\mathrm{M}_{1}$ - With metastases \\
\hline
\end{tabular}

Table 1. Enneking's surgical staging system: $\mathbf{G}_{1}$-Low-grade; $\mathbf{G}_{2}$-High-grade; $\mathbf{T}_{\mathbf{1}}$-Intraosseus; $\mathbf{T}_{2}$-Extraosseus; $\mathbf{M}_{0}$-No metastases; $\mathbf{M}_{\mathbf{1}}$-With metastases.

\section{Staging}

The American National Comprehensive Cancer Network recommends plain radiographs of the lesion and lungs, MRI scan of the extremity, CT scan of the tumor site and of the lungs, and radionuclide bone scan. Technetium Tc-99 methylene diphosphonate scintigraphy will reveal increased metabolic activity at the site of the tumor, but also at the site of distant skip or bone metastases. Thallium (Tl-201) is a potassium analog, actively transported by the sodium-potassium adenosine triphosphatase (ATP) pump. This radioisotope is well accumulated in benign or malignant tumors, reflecting tumor activity. Nevertheless, 
Thallium scanning is mostly used for monitoring the response to neoadjuvant chemotherapy (especially when MRI is not helpful).

Osteosarcoma can be divided into high-grade or low-grade variants, depending of cellularity, pleomorphism, anaplasia and number of mitoses (Fletcher et al. 2002). This fact and the data for presence or absence of osteosarcoma metastases will be enough to do the Enneking's surgical staging (Table 1). This staging system, first used by the American Musculoskeletal Tumor Society and International Symposium on Limb-Salvage is widely accepted. An alternative system, established by the American Joint Committee on Cancer, can be used with Enneking's staging system (Enneking et al., 1980).

\section{Treatment}

A multidisciplinary approach is obligatory in the diagnosis and treatment of osteosarcoma. To achieve high standards in treatment there is a need for specialized radiologists, pathologists, orthopedic and other surgeons (specialized in oncology surgery), pediatric oncologists, specialized physical therapist and often social workers (Wittig et Al., 2002). When a proper chemotherapy and surgery protocol are followed, survival rates surpass $70 \%$. High-grade osteosarcoma patients without clinically detectable lung metastases are presumed to have micro metastases. For these patients treatment consists of preoperative (neoadjuvant) chemotherapy, wide or radical surgical resection and postoperative (adjuvant) chemotherapy i.e. "sandwich therapy". Parosteal osteosarcoma or low-grade intra-medullar osteosarcoma patients are treated with wide or radical surgical resection alone. Chemotherapy is reserved only for cases with high-grade transformation. Periosteal osteosarcoma patients may be treated with preoperative (neoadjuvant) chemotherapy similar to that used for conventional osteosarcomas (Bacci et al., 1993; Bruland \& Phil, 1999).

\subsection{Chemotherapy}

Advances in poly-chemotherapy protocols in the last 30 years have been responsible for improved survival rates and a possibility for limb salvage surgery. Since the beginning of the "odyssey" with Rosen and Jaffe, until now, chemotherapy has been shown to reduce the number of pulmonary metastases or to delay their appearance, facilitating surgical treatment (Rosen et al., 1976).

Standard modern regimens include drugs that have been shown to be the most effective against osteosarcoma: doxorubicin (Adriamycin), cisplatin (Platinol) ifosfamide (Ifex) with mesna (Mesnex) and high-dose methotrexate (Rheumatrex) with Leucoverin calcium rescue. Most standard protocols use doxorubicin and cisplatin with or without high-dose methotrexate for both neoadjuvant (preoperative) and adjuvant (postoperative) chemotherapy. The postoperative (adjuvant) chemotherapy is mostly dependent on the extent of tumor necrosis evaluated after surgical removal. The postoperative chemotherapy regimen is typically the same as the preoperative regimen when tumor necrosis is found to be $\geq 90 \%$ at the time of surgery. "Poor responders" to preoperative chemotherapy, defined as those with $<90 \%$ tumor necrosis at the time of surgery, may benefit from postoperative chemotherapy. In these patients a salvage therapeutic regime is attempted with an increased dose of chemotherapy, an increased length of chemotherapy, or a change in chemotherapeutic agent. Recent trials have incorporated ifosfamide after conventional 
chemotherapeutic drugs to improve patient survival rates (Jaffe et al., 1989; Sæter et al., 1996; Bacci et al., 2001; Messerschmitt et al., 2009).

\subsection{Surgery}

The two primary surgical options are tumor resection with limb-salvage, and amputation. Surgical margins in excision should encompass resection of tumor, pseudo capsule, and a cuff of normal tissue en block. Meticulous preoperative planning before the biopsy and definitive surgery will ensure better results. Prior to the emergence of limb-salvage surgery in the 1970s, amputation of the affected limb was considered the definitive surgical intervention. Amputation remains the indicated treatment when disease-free marginal resection leaves a nonfunctional limb.

The limb-salvage surgery for osteosarcoma patients is possible due to the use of preoperative (neoadjuvant) chemotherapy and to advancement in musculoskeletal imaging, prosthetic implant design and surgical technique (Fig. 5). Today limb sparing surgery is possible for $>85 \%$ of patients with extremities localized osteosarcomas (Bacci et al., 2006; Di Caprio \& Friedlander, 2003; Longhi et al., 2006).

Surgical treatment has to be planed keeping in mind four basic principles of limb-salvage procedures: local recurrence should be no greater and survival no worse than by amputation; the procedure, or treatment of its complications, should not delay adjuvant therapy; reconstruction should be enduring and not associated with a large number of local complications requiring secondary procedures and frequent hospitalizations; function of the limb should approach that obtained by amputation, although body image, patient preference and life style may influence the decision (González-Heranz et al. 1995).

There are a few relative contraindications to be taken in the consideration for limb-salvage surgery: wrong site or ill-planed biopsy; massive encasement of neurovascular bundles; extensive tumour involvement in soft tissue, muscles or skin; complex or complicated (i.e. with infection) pathological fractures; expected inequalities of the extremities more than 8 $\mathrm{cm}$; and exceptionally poor effect of the neoadjuvant chemotherapy. In the process of decision making for limb-salvage surgery versus amputation the "rule of three" can be very helpful. For extremity survival the bone (1), nerves (2), blood vessels (3), and muscle and skin (4) are necessary to be preserved. If osteosarcoma involves one or two of the former structures, limb preserving is possible. If any three of the former are involved, amputation must be taken in consideration (Di Caprio \& Friedlander, 2003).

When "negative" tumor margins are obtained, a large skeletal defect is often present, requiring reconstruction of the bone, muscles, other soft tissues, and the skin. Patient age, tumor location and extent of resection, determine the appropriate surgical alternatives. The extent of the disease, anatomical location of the tumor and the patient's age and psychological profile define the most appropriate surgical procedures. Several options for limb-sparing are available: resection arthrodesis and other similar techniques with special indications (Fig. 7c), modular or special expanding endoprostheses (Fig. 5), corticospongious or bulk auto graft.

For the patients who can't satisfy the principles of limb preservation, ablative surgery has to be taken into consideration. For these patients disarticulation of the hip or shoulder griddle, rotationplasty, femoral or below knee, humeral or other amputations are far more appropriate (González-Heranz et al., 1995; Sæter et al., 1996; Wittig et al., 2002; Samardziski et al. 2009). 


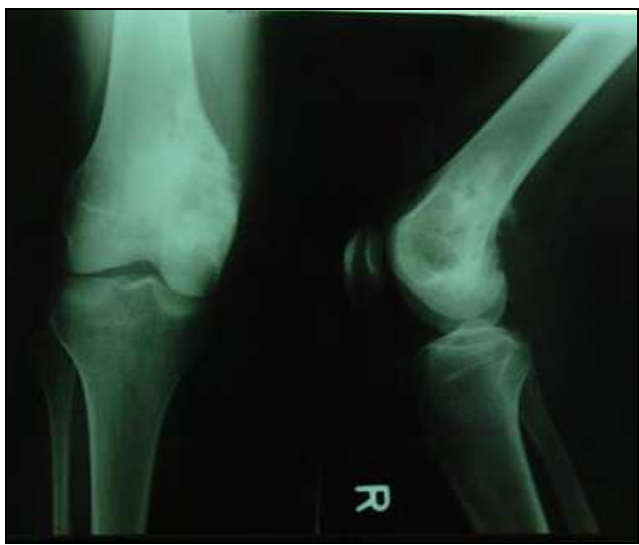

a)

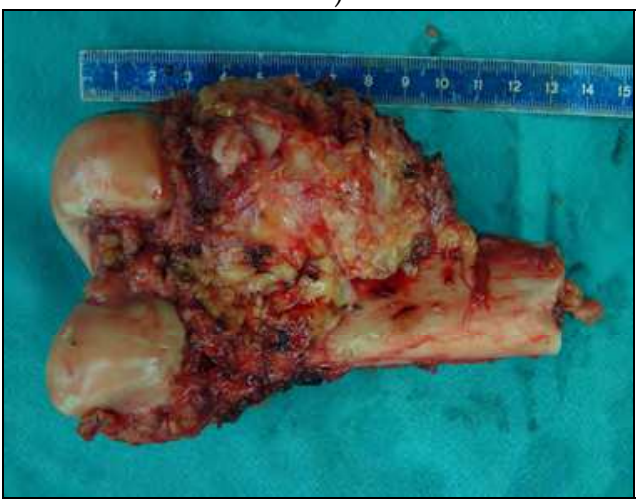

a)

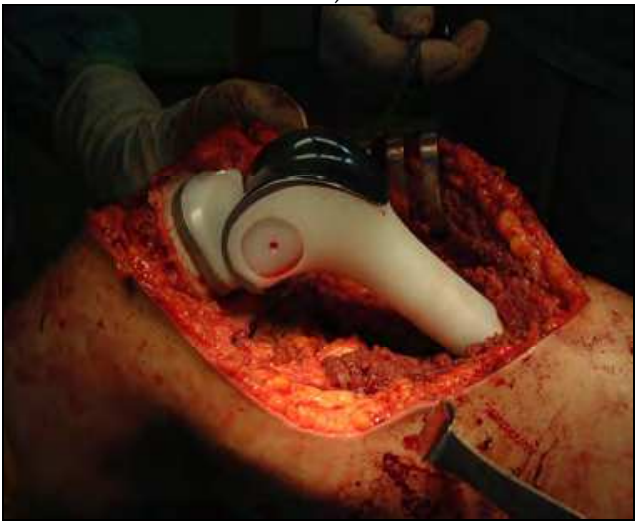

c)

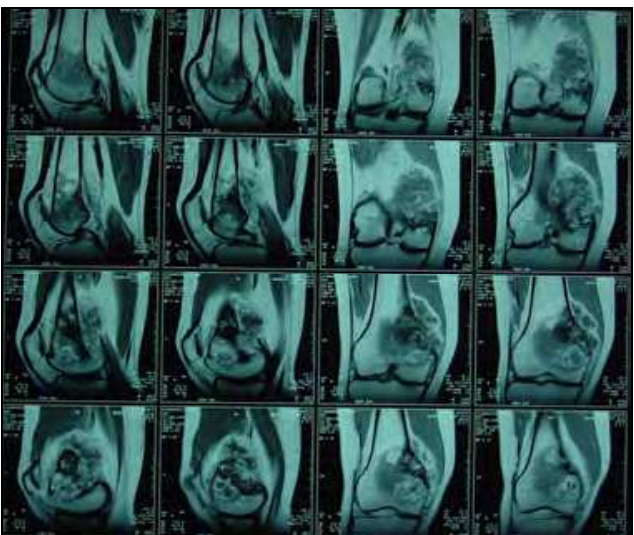

b)

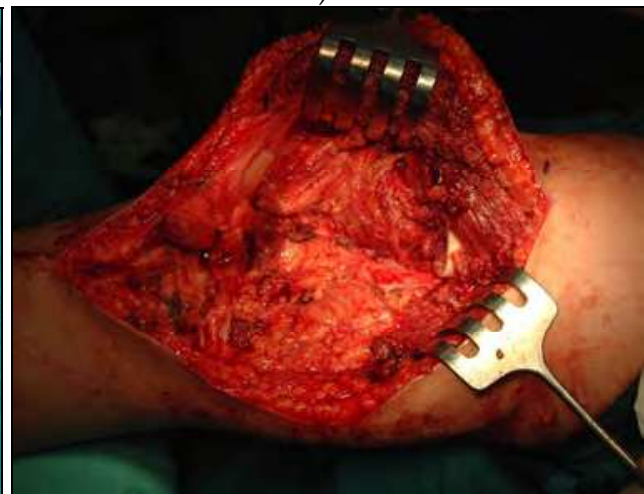

b)

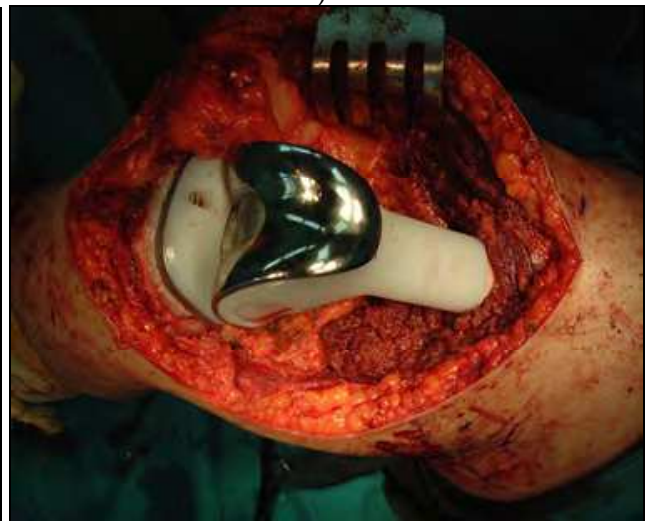

d)

Fig. 5. a) x-ray of high-grade chondroblastic osteosarcoma of right distal femur in a girl of $17 ; \mathbf{b})$ anterior and lateral MRI view of the lesion; c) photo of the resected tumor; d) tumor site ready for reconstruction; $\mathbf{e}, \mathbf{f}$ ) reconstructed right femur and knee (Link modular endoprosthesis). 
The current recommendation for detectable metastases is to excise as many lesions as technically feasible following surgical treatment of the primary tumor. The survival rate for patients can be as high as $60 \%-75 \%$ when both the primary tumor and the solitary lung metastasis are adequately resected (Yonemoto et al., 1997; Bacci et al., 2006).

The rate of surgical site recurrence is $4 \%$ to $6 \%$ for both limb-salvage and amputations. Complications following limb-salvage reconstructions include wound complications, infections, mechanical failure, and nonunion. The reported incidence of complications with limb-salvage surgical techniques is $4 \%$ to $38 \%$ (Kotz et al., 2002).

\subsection{Postoperative follow-up}

After chemotherapy, the patient should be closely followed by the orthopedic oncology surgeon and the medical oncologist. The patient should be monitored for local recurrence, distant or systemic metastases and complications related to reconstruction of the extremity. CT scanning of the chest, plain film radiographs of the reconstructed extremity and meticulous physical examinations are recommended every three months for the first two years and at least every six months from the second year through to the fifth year, and subsequently on a yearly basis. Also, annual bone scintigraphy is mandatory for the first two years after completion of the chemotherapy.

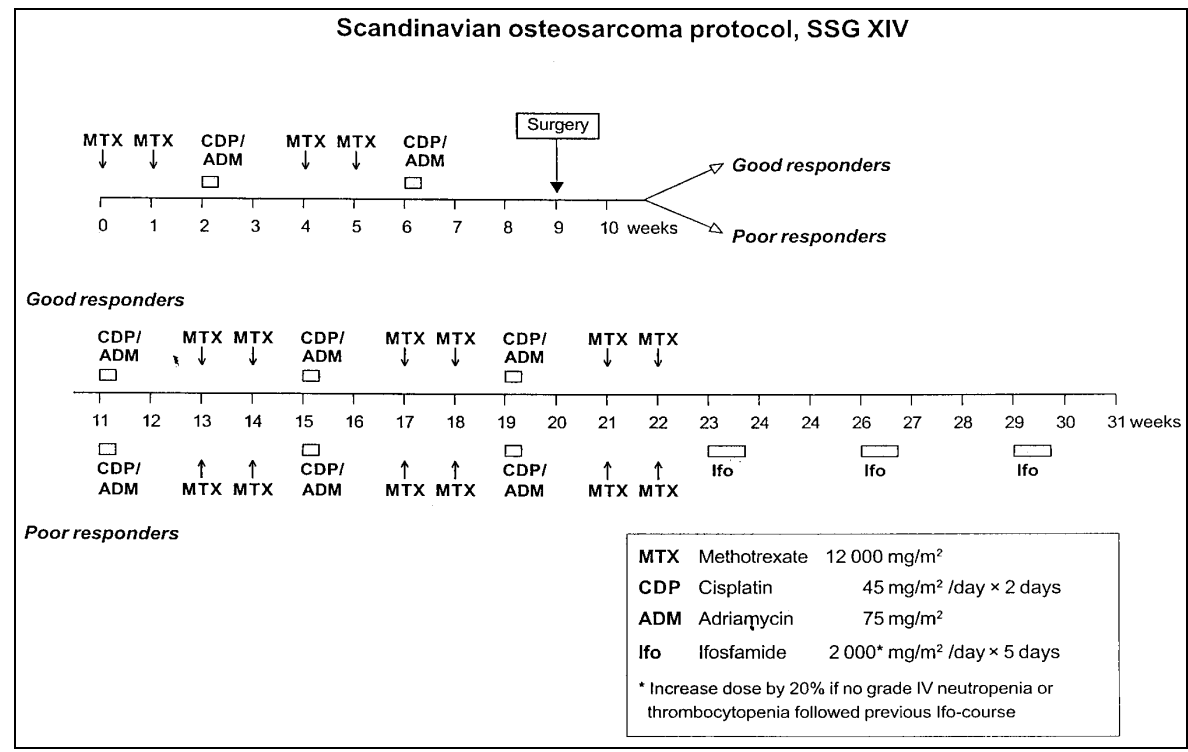

Fig. 6. Scandinavian Sarcoma Group Protocol XIV

\section{Neoadjuvant chemotherapy}

Dramatic changes over the past few decades have occurred with neoadjuvant (preoperative) and adjuvant (postoperative) poly chemotherapy protocols. This improved the ability to perform safe limb-sparing resection of the tumor in more than $85 \%$ of the osteosarcoma patients. Today, as reported in the literature, $60-80 \%$ of the patients with extremity localized non metastatic osteosarcomas are long term survivors. 
Multidrug neoadjuvant chemotherapy, popularized for patients with osteosarcoma by Rosen and later by Jaffe in the late 1970's, is usually initiated as appropriate after histopathological diagnosis and staging. Neoadjuvant chemotherapy protocols with high-dose methotrexate, and cisplatin and doxorubicin dramatically improved long-term survival rates in patients with osteosarcoma sensitive to chemotherapy. Using high dose ifosfamide or different additional and more aggressive therapeutic agents for less sensitive in postoperative chemotherapy (as in: Cooperative Osteosarcomstudiengruppe 96 protocol, Scandinavian Sarcoma Group Protocol XIV and European bone over 40 sarcoma study) improved the results and overall survival of these patients (Kotz et al., 2002). Because of the aggressive nature of the protocols, rescue with Leucoverin (as antidote), bone marrow stimulation with Neupogen and renal protection with Uromitexan are essential. Maximal hydration followed by diuretic forced renal clearance further improves patient's chemotherapy tolerance. During chemotherapy, antiemetics, including: dexamethasone, diphenylhydramine and lorazepam are routinely used in all patients (Bacci et al., 1993; Bruland, 1999; Messershmitt et al., 2009).

\subsection{Various neoadjuvant chemotherapy protocols}

There are various poly-chemotherapy protocols (some in regular practice, other in experimental phase). Basic science is making continuous advance that may yield more specific, less-toxic drugs that will further improve survival rates. The use of high dose Ifosfamide or different, additional and more aggressive, therapeutic agents for less sensitive osteosarcoma patients in postoperative modern chemotherapy becomes a rule. There are many chemotherapy regimens, but most commonly reported are: Cooperative Osteosarcomstudiengruppe 96 protocol (COSS 96), Scandinavian Sarcoma Group Protocol (SSG) XIV, European bone over 40 sarcoma study (EURO-B.O.S.S/COSS), Italian Sarcoma Group protocol (ISG), Sloan-Kettering Center protocol T-10 (SSG III), American Society of Clinical Oncology (ASCO) protocol, etc. Introducing more aggressive chemotherapy for poor responders, improved the results and overall survival of these patients (Brulnad, 1999; Di Caprio \& Friedlander, 2003).

\subsection{Effects of neoadjuvant chemotherapy in high-grade non-metastatic osteosarcoma of extremities}

Various effects of neoadjuvant therapy, such as: remission of pain, reduction of the size of the tumor, sclerosation, pseudo capsule formation, decreasing of neo-vascularisation, tumor necrosis and decrease of the elevated alkaline phosphathase and lactate dehydrogenase levels are widely reported. After neoadjuvant chemotherapy a clinical and radiological response of the tumor has been observed (Bacci et al., 1993). There was reduction, or more often complete remission of pain. This was usually followed with normalization of serum alkaline phosphathase and lactate dehydrogenase levels (if elevated). Bacci further reported an increased density (as seen on Fig. 7b) by the bone lesion on plain radiographs associated with decreased vascularity on angiograms.

Clinical and radiographic reduction in tumor size was observed in more than half of the patients. This was more due to a decrease of the surrounding inflammatory and reactive tissue than to an actual reduction in tumor size. Bacci reported that reduction in vascularity, was the one, most predictable criterion to assess the response of the tumor after neoadjuvant chemotherapy. Neoadjuvant chemotherapy may also decrease the size of the primary tumor 
(Fig. 7) by reducing its neo-vascularity and promoting tumor demarcation from surrounding tissue with pseudo-capsule (Fig $5 \mathrm{~b}$ ). This makes limb-salvage surgery technically more feasible, even if a marginal resection is obtained (Messershmitt et al., 2009).

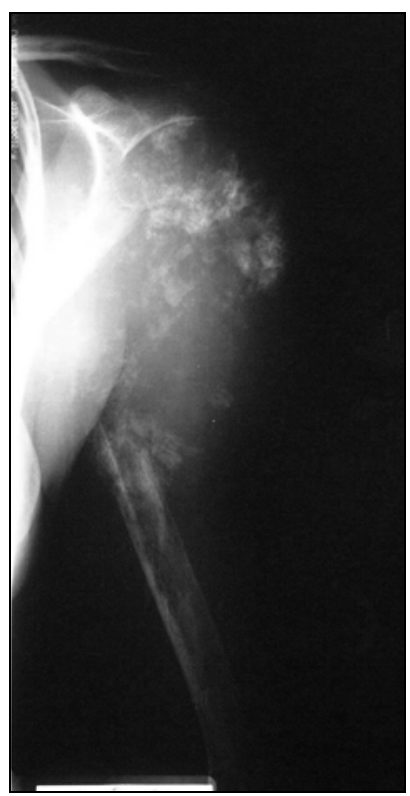

a)

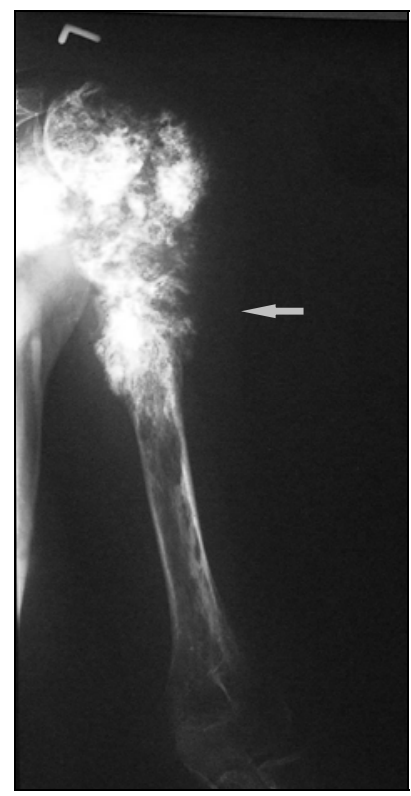

b)

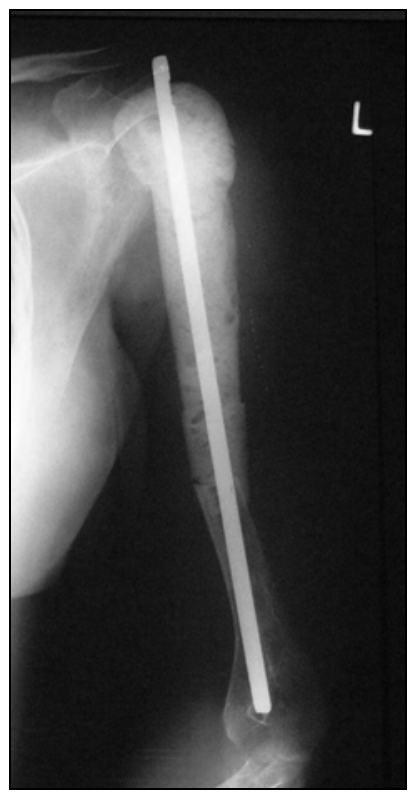

c)

Fig. 7. a) Fifteen years old female osteosarcoma patient with pathological fracture of the left proximal humerus at the first presentation. The patient had preoperative (neoadjuvant) chemotherapy with Swedish Sarcoma Protocol XIV. b) Excellent response (>90\% tumor necrosis) with sclerosation after neoadjuvant chemotherapy (arrow shows the site of the pathological fracture). c) Radiograph of the humerus after wide resection of the osteosarcoma, and first stage reconstruction of the bone with intramedullary rod and bone cement.

The primary goal of neoadjuvant chemotherapy is to treat undetectable (or micro) metastases. It is reasonable to believe that neoadjuvant chemotherapy may decrease the risk of spreading viable tumor cells after biopsy, and therefore, decrease the possibility of distant metastases and of local recurrence. This is only possible with optimal serum concentration of methotrexate (at least $1000 \mu \mathrm{M}$ ) at the end of a 6 hour infusion. All of these advantages of neoadjuvant chemotherapy enables more options for wide or near-marginal resection of the tumor and for a limb-sparing surgery (Bruland \& Phil, 1999).

\subsection{Macedonian long-term follow-up experiences with the effects of neoadjuvant chemotherapy in patients with extremity localized high-grade osteosarcoma}

Following the "wave of modern" poly-chemotherapy, in the period 2000-2008, a prospective study was done at the University Clinic for Orthopedic Surgery and Institute of Radiology 
and Oncology in Skopje. In this period, 47 patients with high-grade osteosarcoma, were treated (Samardziski et al., 2009a).

Selection of patients for neoadjuvant chemotherapy and limb-salvage surgery was based on the following criteria:

Inclusion criteria:

- $\quad$ histopathologically proven high-grade osteosarcoma (grade III or IV);

- primary localization on the extremities, with no evidence of lung or other metastases;

- $\quad$ patient age between 8 and 65 years; normal hepatic and renal function;

- leukocyte count over $3.0 \times 109 / \mathrm{L}$ and platelet count over $100 \times 109 / \mathrm{L}$;

- neoadjuvant chemotherapy was introduced no longer than 1 month after histological diagnosis of osteosarcoma.

Exclusion criteria:

- $\quad$ patients with central localization of osteosarcoma (e.g. pelvis, vertebra);

- $\quad$ evidence of lymphatic or haematogenous metastases at the time of diagnosis;

- patients under 8 years or older than 65 years;

- pregnant or nursing women.

According to the exclusion criteria, 8/47 patients were excluded, owing to lung metastases at first presentation or pelvic localization. Another 10/39 patients were excluded from the study due to primary indication for ablative surgery (amputation or disarticulation). Seventy five percent of the patients (29/39) were treated with limb-sparing surgery (Table 2). Fourteen $(48 \%)$ patients were male and $15(52 \%)$ were female. The mean age was $23.4 \pm 14.5$ years (range 8-63). Mean follow-up was $49.9 \pm 23.1$ months (range 23-108).

All patients received to the Scandinavian Sarcoma Group XIV neoadjuvant chemotherapy protocol (SSG XIV). Patients received 2 cycles of preoperative chemotherapy (high dose methotrexate $1200 \mathrm{mg} / \mathrm{m}^{2}$, cisplatin $45 \mathrm{mg} / \mathrm{m}^{2} /$ day $\times 2$ days, and doxorubicin $75 \mathrm{mg} / \mathrm{m}^{2}$ ), (Fig. 6).

After resection, a detailed histopathological assessment of the specimen was done to determine the extent of necrosis of the tumor tissue. Considering the percentage of necrotic tumor tissue, patients were classified into two groups. The first group experienced good response to chemotherapy ( $>90 \%$ necrosis of the tumor). The second group had a poor response to chemotherapy ( $>10 \%$ viable tumor). Regarding good or poor response of the tumor to chemotherapy, patients followed different branches of the protocol (Fig. 6). All 29 patients received 3 courses of postoperative chemotherapy (the same as preoperative). Patients with poor response received 3 more cycles of chemotherapy with high dose ifosfamide $\left(2000 \mathrm{mg} / \mathrm{m}^{2} /\right.$ day $\times 5$ days plus Mesna) every 3 weeks (Fig. 6). Histopathological assessment of the specimen did not only identify the extent of tumor necrosis, but information on tumor-free margins, too.

We have analyzed the following parameters of the clinical and radiological data after neoadjuvant chemotherapy: age, gender, time of follow-up, necrosis of the resected tumor after neoadjuvant chemotherapy (poor or good response), decrease of pain, decrease in tumor diameter, tumor pseudo-capsule seen on MRI, sclerosis seen on radiographs or CT, local recurrence and metastases (Table 2).

Response to neoadjuvant chemotherapy was good (more than $90 \%$ necrosis of the tumor) in $16 / 29$ patients $(55.2 \%)$. The examinees with good response to neoadjuvant therapy had significantly longer overall survival time than the patients with poor response (fig. 8). Ten percents of the patients with poor response survived for more than 65 months, while $58 \%$ of the patients with good response survived for more than 100 months (Log-Rank test $=3,74 \mathrm{p}=0.0002)$. 


\begin{tabular}{|c|c|c|c|c|c|c|c|c|c|c|c|}
\hline $\begin{array}{c}\text { Patie- } \\
\text { nt } \\
\text { No. }\end{array}$ & $\begin{array}{c}\text { Age } \\
\text { (y.) }\end{array}$ & Gender & $\begin{array}{c}\text { Follow- } \\
\text { up (m.) }\end{array}$ & $\begin{array}{c}\text { Response } \\
\text { to neoa-d. } \\
\text { chemoth. }\end{array}$ & $\begin{array}{c}\text { Decrease } \\
\text { of pain }\end{array}$ & $\begin{array}{c}\text { Decrease } \\
\text { in } \\
\text { diameter }\end{array}$ & $\begin{array}{c}\text { Pseudo- } \\
\text { capsule }\end{array}$ & $\begin{array}{c}\text { Sclerosis } \\
\begin{array}{c}\text { Recurre- } \\
\text { nce } \\
\text { (m.) }\end{array}\end{array} \begin{array}{c}\text { Metasta- } \\
\text { ses } \\
\text { (m.) }\end{array}$ & $\begin{array}{c}\text { Deceased } \\
\text { after } \\
\text { (m.) }\end{array}$ \\
\hline 1 & 25 & M & 30 & P & 0 & 1 & 0 & 0 & 0 & 22 & 30 \\
\hline 2 & 13 & M & 32 & P & 1 & 1 & 1 & 0 & 0 & 27 & 32 \\
\hline 3 & 23 & M & 50 & G & 1 & 1 & U & 1 & 0 & 29 & - \\
\hline 4 & 16 & F & 44 & P & 1 & 1 & 1 & 1 & 0 & 38 & 44 \\
\hline 5 & 15 & F & 68 & G & 1 & 1 & 1 & 1 & 50 & 57 & 68 \\
\hline 6 & 14 & M & 51 & G & 1 & 1 & 1 & 1 & 0 & 0 & - \\
\hline 7 & 8 & F & 50 & G & 1 & 1 & 1 & 1 & 29 & 43 & 50 \\
\hline 8 & 13 & M & 45 & G & 1 & 1 & 1 & 1 & 0 & 0 & - \\
\hline 9 & 16 & F & 54 & P & 1 & 1 & 1 & 1 & 0 & 36 & 54 \\
\hline 10 & 17 & F & 23 & P & 0 & 0 & 0 & 0 & 6 & 12 & 23 \\
\hline 11 & 54 & F & 38 & P & 1 & 1 & U & 1 & 0 & 0 & 38 \\
\hline 12 & 14 & F & 98 & G & 1 & 1 & 1 & 1 & 0 & 0 & - \\
\hline 13 & 63 & M & 106 & G & 1 & 1 & 1 & 1 & 96 & 100 & 106 \\
\hline 14 & 17 & M & 67 & P & 1 & 0 & 1 & 1 & 54 & 60 & 67 \\
\hline 15 & 16 & M & 59 & G & 1 & 1 & 1 & 0 & 0 & 0 & - \\
\hline 16 & 20 & F & 54 & P & 1 & 1 & 1 & 0 & 0 & 46 & 54 \\
\hline 17 & 20 & F & 47 & G & 1 & 1 & 1 & 1 & 0 & 0 & - \\
\hline 18 & 16 & M & 10 & P & 0 & 0 & 0 & 0 & 2 & 4 & 10 \\
\hline 19 & 39 & F & 61 & P & 1 & 0 & 1 & 1 & 53 & 57 & 61 \\
\hline 20 & 14 & M & 26 & P & 0 & 0 & 0 & 0 & 19 & 19 & 26 \\
\hline 21 & 8 & M & 40 & G & 1 & 1 & 1 & 0 & 0 & 0 & - \\
\hline 22 & 44 & F & 59 & G & 1 & 1 & U & 1 & 0 & 0 & - \\
\hline 23 & 14 & M & 40 & G & 1 & 1 & 1 & 1 & 0 & 30 & 40 \\
\hline 24 & 44 & F & 35 & P & 0 & 1 & 0 & 0 & 21 & 28 & 35 \\
\hline 25 & 15 & F & 108 & G & 1 & 1 & 1 & 0 & 0 & 0 & - \\
\hline 26 & 15 & M & 27 & P & 0 & 0 & 0 & 0 & 2 & 11 & 27 \\
\hline 27 & 48 & F & 43 & G & 1 & 1 & 1 & 1 & 0 & 0 & - \\
\hline 28 & 24 & F & 33 & G & 1 & 1 & 1 & 1 & 18 & 0 & - \\
\hline 29 & 34 & M & 51 & G & 1 & 1 & 0 & 1 & 35 & 45 & 51 \\
\hline
\end{tabular}

M: male; F: female; G: good response after neoadjuvant chemotherapy (necrosis $>90 \%$ of the tumor); $\mathbf{P}$ : poor response after neoadjuvant chemotherapy (>10\% viable tumor); $\mathbf{U}$-unknown or missing data; 1yes; 0 -no or none.

Table 2. Clinical data of patients with high-grade osteosarcoma of the extremities, treated with neoadjuvant chemotherapy.

Local recurrence appeared in 17/29 patients (58.6\%). The examinees without local relapse had significantly longer overall survival time than the examined persons with no relapse. Ten percent of the patients with relapse survived more than 100 months, while $48 \%$ of the examined with no local relapse were alive even after 100 months (Log-Rank test $p=0.0002$ ). 
Most of the tumor relapses were seen in the patients by 22 months after surgery. The 3 patients with early local recurrences had secondary extirpation of the relapsed tumor and one of them had to be amputated.

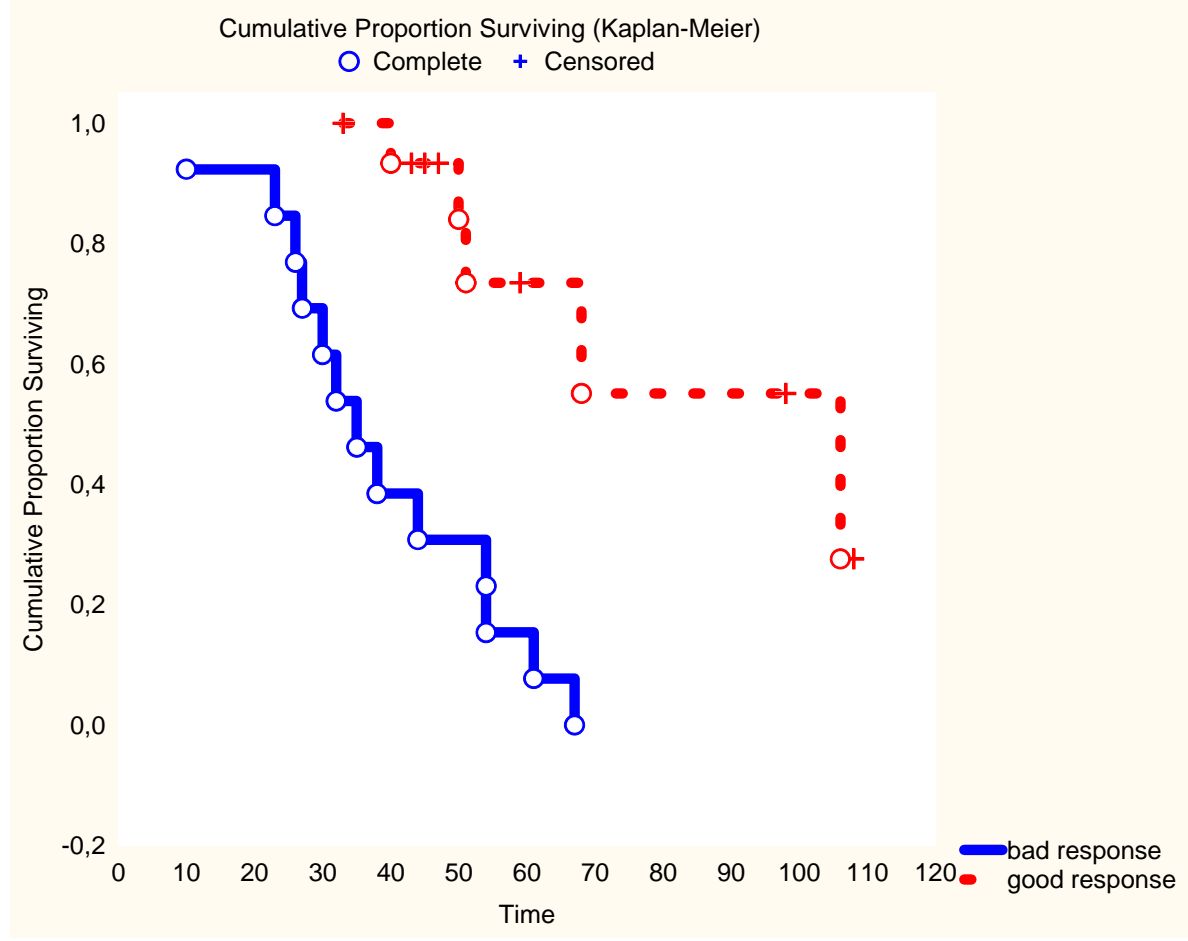

Fig. 8. Response of the patients after neoadjuvant chemotherapy treated with SSG XIV chemotherapy protocol.

Lung metastases appeared in $18 / 29$ patients or $62.1 \%$. The examinees with metastases had significantly shorter overall survival time than the metastasis-free patients. Four percent of the examined patients with metastases survived longer than 100 months, while $90 \%$ of the examined with no metastases were alive even after 100 months (Log-Rank test $\mathrm{p}=0.0002)$.

Plain radiograph or CT-scan sclerosis of the tumor after neoadjuvant chemotherapy was seen in 18/29 patients (62.1\%). Pseudo-capsule was seen in 19/29 patients (65.5\%), but in $3 / 29(10.3 \%)$ MRI imaging showed inconclusive data. Cystic necrosis after neoadjuvant chemotherapy was seen in $14 / 29$ patients (48.3\%). Inconclusive results for cystic necrosis were found in 3 and data was missing for 1 patient.

Up to date $10 / 29$ patients $(34.5 \%)$ are disease or event free. Mean survival time of the patients was 53 months, and $20 \%$ of the examinees survived longer than 60 months (Fig. 9). 


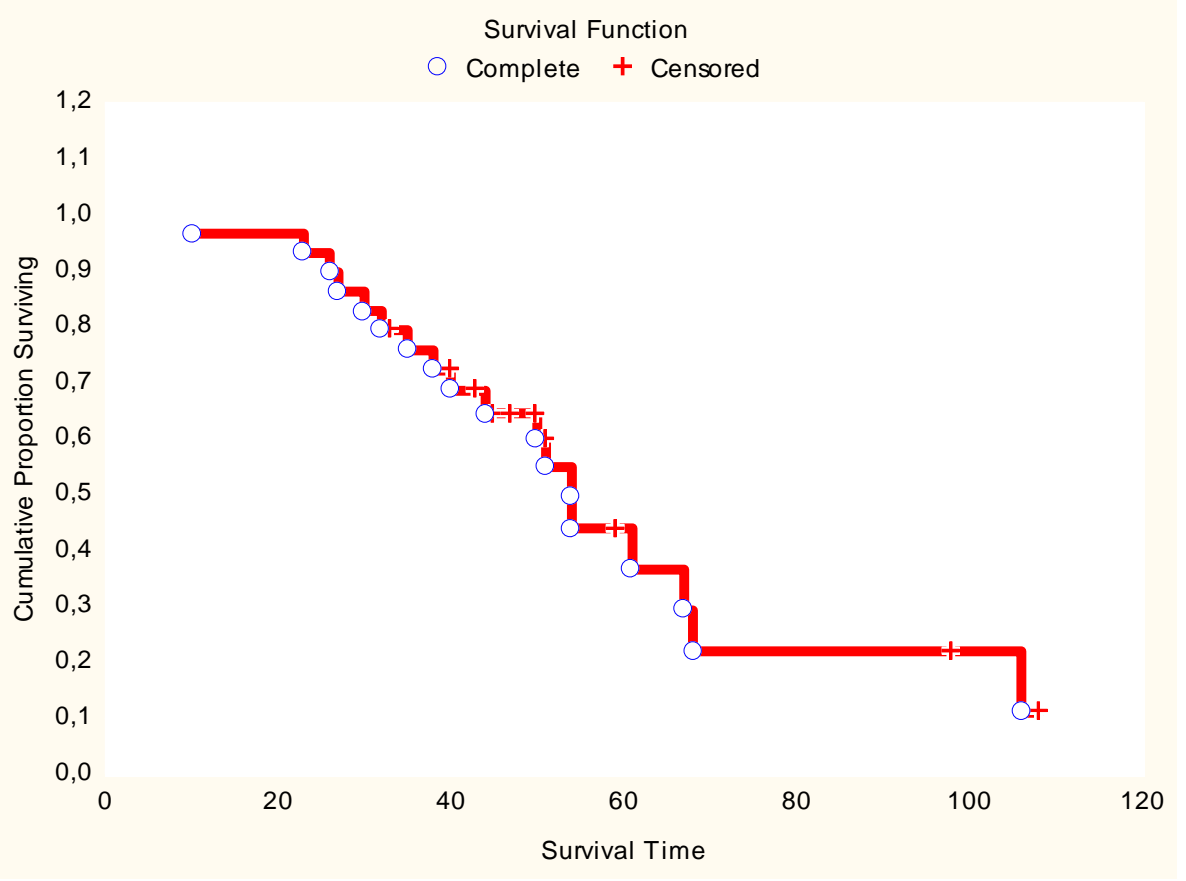

Fig. 9. Disease and event free survival time of the patients with extremity localized highgrade osteosarcoma treated with SSG XIV neoadjuvant chemotherapy protocol and surgery.

Using high dose ifosfamide for poor responders in postoperative chemotherapy should improve the results and overall survival time of these patients. If treatment and management principles of high-grade osteosarcoma are followed, limb-sparing with $60-80 \%$ survival rates could be achieved. Our preliminary results are slightly different from those published in the literature. There was a significantly different overall survival time in our study between the group of patients with good response to neoadjuvant chemotherapy compared to the group of patients with bad response. Furthermore, overall survival time in our group of patients was shorter than the time reported in the literature. In spite of the recorded differences in the results, the treatment regimen with neoadjuvant chemotherapy is promising and encouraging.

\subsection{Toxic effects of neoadjuvant chemotherapy regimen}

Most often hematologic toxicity is seen after chemotherapy. Various authors report Grade 3 or Grade 4 hematologic toxicity in $10-15 \%$ of the treated patients. Severe leucopenia and/or thrombocytopenia are the two conditions for readmitting patients in hospital. In that case Neupogen or Leucoverin rescue treatment is beneficial (Bacci \& Picci, 1994).

Most of these patients with myelotoxicity have fever and microbiologically proven bacteremia during their granulocytopenic phase. Wide spectrum antibiotics in the beginning and specific antibiotics after microbiological assessment are necessary. 
Cardiotoxicity is less often reported in patients with neoadjuvant chemotherapy. Unfortunately this side effect of the treatment is most serious and cardiopathy following the treatment may become a chronic life treating condition.

Due to general toxic effects to the human body and profound systemic reaction of all organs and systems to chemotherapeutics, sickness, malaise and weakness are general side effects. A common problem is abdominal pain associated with mild asscites, easily visualised on ultrasonography. Therefore antiemetics and corticosteroids, including dexamethasone, diphenylhydramine and lorazepam are routinely used in all patients (Bacci et al., 1993; Bruland, 1999; Messershmitt et al., 2009).

Skin necrosis, tender local swelling, inflammation, due to local intra-arterial cisplatin or venous trombophlebitis occurres in some patients. This lesions usually do not cause a major problem and usually heal in 2-3 weeks with pigmented scars.

\section{Discussion}

Prior to the introduction of chemotherapy, when amputation was the primary treatment for patients with osteosarcoma, the predicted long-term survival was 15-20\%. Dismal survival rates were presumably attributable to pulmonary metastatic disease, whether clinically obvious or occult (Enneking, 1975). Survival rates dramatically increased during 1970's and 1980 's with the pioneering work of Rosen and Jaffe. Currently, long-term survival rates are $60 \%$ to $70 \%$ for patients with localized osteosarcoma and for extremity localized up to $80 \%$ (Meyers et al., 2008). Despite the use of modern neoadjuvant chemotherapy the 10-year survival rates decline significantly to $20 \%$ in patients with clinically detectable metastases. Most of the patients ultimately die because of respiratory failure caused by the metastatic burden (Bacci et al., 2008; Messerschmitt et al., 2009; Samardziski et al., 2009a). Excluding high-grade surface osteosarcoma, which has similar prognosis to that of conventional osteosarcoma, the surface (parosteal and periosteal) osteosarcoma variants have the best prognosis of all. The 10-year survival rates for this group of patients is up to $85 \%$ (Samardziski et al., 2009b).

The site of the lesion has prognostic importance. The best survival rates are expected in patients with appendicular localization of the osteosarcoma. Central localization (pelvis, ribs and vertebrae) are less common sites of osteosarcoma, and have poorest prognosis. Osteosarcoma of the jaw is associated with an especially good prognosis, whereas osteosarcoma involving the skull has a very poor prognosis (Unni, 1998; Yu \& Wang, 2009). Badly planned and ill preformed biopsy can complicate the final surgery and may decrease survival rates due to local spreading or risk for early metastatic disease (Mankin et al., 1982; Campanacci, 1999).

The overall treatment results in high-grade osteosarcoma are less impressive than widely presumed. Whereas classical osteosarcoma survival has indeed increased, in other subgroups, comprising more than $40 \%$ of the entire osteosarcoma population, the prognosis has been modestly improved. Today still more than half of an unselected osteosarcoma population eventualy succumbs to the disease, despite the current multimodal primary treatment as well as second-line chemotherapy and surgical metastatectomies (Bruland, 1999).

Neoadjuvant chemotherapy enables limb-sparing in the majority of patients with extremity localised ostesarcoma. During the past 20 years dramatic advances have been made in the 
treatment of non-metastatic osteosarcoma in terms of cure rate and quality of life for survivors. These advances are due mainly to the development of effective adjuvant and neoadjuvant chemotherapeutic regimens. Reports on the progress and controversies in the treatment of osteosarcoma occurred with respect to the construct, expirimental design and interpretation of the studies. Never the les, this sdudyes led to remarkable results (Bacci, 2008).

\subsection{Prognosis}

Poor prognostic factors for patients with osteosarcoma include metastases at first presentation, extremely large primary tumor, increased alkaline phosphatase and lactate dehydrogenase levels, poor response to neoadjuvant chemotherapy, tumor discontinuous from bone, pathologic fractures and lymph node involvement (Longhi, et al. 2006). Despite current surgical and chemotherapeutic treatment regimens, $30 \%$ to $40 \%$ of osteosarcoma patients experience relapse within 3 years of treatment. Pulmonary recurrence is most common secondary to micro-metastatic disease. Regardless of poor prognosis, repeated tumor excisions can be performed (of primary site or metastatic one), because many studies have shown improved survival rates (Yonemoto, 1997; Bacci et al., 2001). The role of "second-line" chemotherapy regimen remains controversial because no standard regimen exists for the recurrence of the tumor.

The evaluation variables influencing systemic and local recurrence and final outcome are extremely important in defining risk-adapted treatments for patients with nonmetastatic osteosarcoma of the extremity. Upon multivariate analysis, age $\leq 14$ years, high serum levels of alkaline phosphatase, tumor volume $>200 \mathrm{~mL}$, a dual-drug regimen chemotherapy, inadequate surgical margins, and poor histologic response to treatment maintained independent prognostic values on the outcome of nonmetastatic osteosarcoma of the extremities. These factors must be considered when deciding risk-adapted treatments for osteosarcoma patients (Pochanugool, 1997; Bacci, 2006; Yu \& Wang, 2009). Amputation remains the indicated treatment when these factors are taken into consideration or tumor resection to disease-free margins leaves a nonfunctional limb (Enneking, 1975; Di Caprio \& Friedlander, 2003).

\subsection{Future considerations}

A logical development of chemotherapy was introduction of local (or loco-regional) intraarterial methods of chemotherapy. The obvious limitations are complicated intra-arterial techniques of application of chemotherapeutics and uncontrolled risk of tissue necrosis. Intra-arterial administration of cisplatin has been investigated for achieving improved histological response following chemotherapy. Since the originalr attempts to administer intra-arterial cisplatin from the $1980^{\prime}$ s, major advance in imaging and surgical techniques have improved the results and made it easier and safer. Reported studies demonstrate an increase in long-term survival up to $93 \%$. Thus, a consensus on the routine use of intraarterial chemotherapy does not exist (Jaffe, 1989; Bacci et al., 2001; Messershmit et al., 2009). Basic science is making continuous advance in molecular mechanisms and biologic pathways that may yield more specific, less-toxic drugs that will further improve survival rates. Inhibition of tyrosine kinase signaling is known to regulate cell growth, cell proliferation, angiogenesis, and apoptosis and is an area of current interest (Messershmit et al., 2008). Liposomal muramyl tripeptide phosphatidilethanolamine (L-MTP-PE) is a 
promising drug in clinical trial that functions to stimulate the formation of tumoricidal macrophages (Meyers et al., 2008).

\section{Conclusion}

With advances in neoadjuvant chemotherapy, radiographic imaging, and reconstructive surgery, most patients with osteosarcoma can now be offered limb-sparing treatment. A multidisciplinary approach in diagnosis and treatment is mandatory. Surgical resection with wide margins after neoadjuvant and adjuvant chemotherapy after surgery is a current standard of care. Osteoarticular allografts, modular prostheses, or composites of these two approaches form the basis for most current reconstructions. However, amputation still plays an important role and offers a standard to which other approaches must be compared. Basic science is making continuous advance in molecular mechanisms and more specific, lesstoxic drugs will further improve survival rates. Current research into the cell biology of osteosarcoma may lead to improved and more target-selective treatment with the intent of improved overall survival.

Applying neoadjuvant chemotherapy followed by appropriate surgery requires responsible, trained and highly engaged medical staff. If treatment and management principles of highgrade osteosarcoma with neoadjuvant therapy are followed, long-term 60-80\% overall survival rates could be easily achieved.

\section{Acknowledgement}

Special thanks to Mrs. Marija Tanevska-Pulios for English language editing of the paper.

\section{References}

Bacci, G. et al. (1993). Primary chemotherapy and delayed surgery for non-metastatic osteosarcoma of the extremities. Cancer, Vol. 72, No.11, pp. (3227-3238), DOI: 10.1002/1097-0142(19931201)72:11<3227::AID-CNCR2820721116>3.0.CO;2-C.

Bacci, G. \& Picci, P. (1994). Analysis of Factors Influencing Treatment Options in Osteosarcoma. Review. Forum, Vol. 4, No.1,(1994), pp. (52-64).

Bacci, G. et al. (2001). A comparison of methods of loco-regional chemotherapy combined with systemic chemotherapy as neoadjuvant treatment of osteosarcoma of the extremity. Eur J Surg Oncol, Vol. 27, (2001), pp. (98-104), PMID: 11237499.

Bacci, G. et al. (2006). Prognostic factors for osteosarcoma of the extremity treated with neoadjuvant chemotherapy: 15-Year experience in 789 patients treated at a single institution. Cancer, Vol. 106, No. 5, (2006), pp. (1154-1161), ISSN 0008543 X.

Bacci, G. et al. (2008). High-grade osteosarcoma of the extremities with lung metastases at presentation: Treatment with neoadjuvant chemotherapy and simultaneous resection of primary and metastatic lesions. J Surg Oncol, Vol. 98, (2008), pp. (415420) PMID: 18792969.

Bruland, ØS. \& Phil, A. (1999) On the current management of osteosarcoma. A critical evaluation and a proposal for modified treatment surgery. Eur J Cancer, Vol. 33, pp. (1725-1731), PMID: 9470825. 
Campanacci, M. (1999). Errors in the diagnosis and treatment of the musculoskeletal tumors: What must not be done. Chir Organi Mov, Vol. 84, (1999), pp. (1-17).

Di Caprio, MR. \& Friedlander, GE. (2003). Malignant Bone Tumours: Limb Sparing Versus Amputation. J Am Acad Ort Surg, Vol.11, No.1, pp. (125-129), PMID: 12699369.

Enneking, WF. (1975). Osteosarcoma (editorial comment). Clin Orthop Rel Research, Vol. 111, (1975), pp. (2-4).

Enneking, WF. et al. (1980). A surgical staging of musculoskeletal sarcomas. J Bone Joint Surg (Am), Vol. 62, (1980), pp. (1027-1030), PMID: 7449206.

Fletcher, CDM. et al. (2002). Classification of Tumours. Pathology and Genetics of Tumours of Soft Tissue and Bone. IARC Press, Lyon, ISBN: 9283224132.

González-Heranz, P. et al. (1995). The Management of Limb-Length Discrepancies in Children after Treatment of Osteosarcoma and Ewing's Sarcoma. J Ped Orthop, Vol. 15, (1995), pp.(561-565), PMID: 7593562.

Jaffe, N. et al. (1985). Analysis of efficacy of the intra-arterial cis-Diamminedichlorplatinum-II and high dose methotrexate with citrovorum factor rescue in the treatment of primary osteosarcoma. Reg Cancer Treat, (1985). Vol. 2, pp. (15763), PMID: 3874932.

Kotz, R. et al. (2002). Advances in bone tumor treatment in 30 years with respect to survival and limb salvage. A single institution experience. Intern Othoped, Vol. 26, (2002), pp. (197-206), DOI: 10.1007/s00264-002-0365-1.

Lodwick, GS. et al. (1963). Computer Diagnosis of Primary Bone Tumours, A Preliminary Report, J Bone Joint Surg Am, Vol. 80. (1963), pp. (273-275), DOI: 10.1148/80.2.273.

Longhi, A. et al. (2006). Primary bone osteosarcoma in the pediatric age: State of the art. Cancer Treat Rev,Vol. 32, (2006), pp. (423-436), DOI:10.1016/j.ctrv.2006.05.005.

Mankin, HJ. et al. (1982). The Hazards of Biopsy in Patients with Malignant Primary Bone and Soft-Tissue Tumours. J Bone Joint Surg Am, Vol. 64 (1982), pp. (1121-1127), PMID: 7130225.

Messershmitt, PJ. Et al. (2008). Specific tyrosine kinase inhibition regulated human osteosarcoma cells in vitro. Clin Orthop Rel Res, Vol. 466, (2008), pp. (2168-2175), DOI: 10.1007/S11999-008-0338-9.

Messerschmitt, PJ. et al. (2009). Osteosarcoma. J Am Acad Ort Surg,; Vol. 17, (2009), pp. (515-527), PMID: 19652033.

Meyers, PA. et al. (2008). Osteosarcoma: The addition of muramyl tripeptide to chemotherapy improves overall survival. A report from Children's Oncology Group. J Clin Oncol, Vol. 26, (2008), pp. (633-638), PMID: 18235123.

Price, CH. \& Jeffree, GM. (1977). Incidence of bone sarcoma in SW England, 1946-74, in relation to age, sex, tumour site and histology. Br J Cancer. Vol.36, No.4, (October 1977), pp. (511-522), PMCID: PMC2025376.

Pochanugool, L. et al. (1997). Prognostic Factors Among 130 Patients With Osteosarcoma. Clin Orthop Rel Res. (1997). Vol. 345, pp.(206-214), PMID: 9418642.

Rosen, G et al., (1976). Chemotherapy, en bloc resection, and prosthetic bone replacement in the treatment of osteogenic sarcoma. Cancer, Vol. 37, No.1, (1976), pp. (1-11), PMID: 1082364. 
Sæter, G. et al. (1996), Extremity and non-extremity high-grade osteosarcoma, Acta Oncol, Vol. 35, (1996), pp. (129-134), PMID: 9073059.

Samardziski, M. et al. (2004). Computer assisted diagnosis of benign bone tumors. Radiol Oncol,Vol. 38, (2004), pp. (165-169), ISSN: 1581-3207.

Samardziski, M. et al. (2009a). Limb-sparing in patients with non-metastatic high-grade osteosarcoma. J. BUON, Vol. 14, (2009) pp. (63-69), PubMed ID: 19373949.

Samardziski, M. et al. (2009b). Parosteal Osteosarcoma. Bratisl Lek Listy. Vol. 110, (2009), pp. (240-244), PubMed ID: 19507652.

Unni, KK. (1998). Osteosarcoma of bone. J Orthop Sci, Vol. 3, (1998), pp. (287-294), DOI: $10.1007 / \mathrm{s} 007760050055$.

Wittig, JC. et al. (2002). Osteosarcoma: A Multidisciplinary Approach to Diagnosis and Treatment. Am Fam Physician, Vol. 65, No. 6, (2002), pp. (1123-1132), PMID: 11925089.

Yonemoto, T. et al. (1997). Prognosis of Osteosarcoma with Pulmonary Metastases at Initial Presentation is not Dismal. Clin Orth Rel Res, Vol. 349, (1997), pp. (194-199), PMID: 9584383.

$\mathrm{Yu}, \mathrm{XC}$ \& Wang, W. (2009). Multivariate analysis of factors influencing on preoperative chemotherapy response for osteosarcoma. Cancer Research on Prevention and Treatment, Vol. 36, No.10, (2009) pp. 863-868, ISSN 1000-8578. 


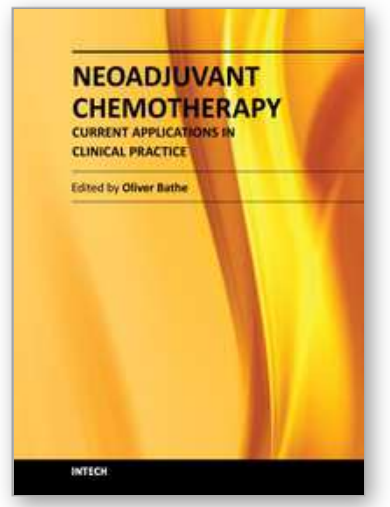

\author{
Neoadjuvant Chemotherapy - Current Applications in Clinical \\ Practice \\ Edited by Dr. Oliver Bathe
}

ISBN 978-953-307-994-3

Hard cover, 268 pages

Publisher InTech

Published online 01, February, 2012

Published in print edition February, 2012

The most significant advances in cancer therapy in recent years have involved the development of systemic therapeutics. With improvements in response rates in solid tumors, opportunities have arisen to enhance the effectiveness of surgery. Administration of systemic therapy prior to surgery - neoadjuvant chemotherapy represents one approach by which clinicians have successfully reduced the extent of surgery and, in some instances, positively impacted on clinical outcomes. This collection of works by expert clinicians from a variety of disciplines represents an exploration of the current knowledge of the role of neoadjuvant chemotherapy in diverse tumor types.

\title{
How to reference
}

In order to correctly reference this scholarly work, feel free to copy and paste the following:

Milan Samardziski, Vesna Janevska, Beti Zafirova-Ivanovska, Violeta Vasilevska and Slavica Kraleva (2012). Effects of Neoadjuvant Chemotherapy in High-Grade Non-Metastatic Osteosarcoma of Extremities, Neoadjuvant Chemotherapy - Current Applications in Clinical Practice, Dr. Oliver Bathe (Ed.), ISBN: 978-953307-994-3, InTech, Available from: http://www.intechopen.com/books/neoadjuvant-chemotherapy-currentapplications-in-clinical-practice/effects-of-neoadjuvant-chemotherapy-in-high-grade-nonmetastaticosteosarcoma-of-the-extremities

\section{INTECH}

open science | open minds

\author{
InTech Europe \\ University Campus STeP Ri \\ Slavka Krautzeka 83/A \\ 51000 Rijeka, Croatia \\ Phone: +385 (51) 770447 \\ Fax: +385 (51) 686166 \\ www.intechopen.com
}

\author{
InTech China \\ Unit 405, Office Block, Hotel Equatorial Shanghai \\ No.65, Yan An Road (West), Shanghai, 200040, China \\ 中国上海市延安西路65号上海国际贵都大饭店办公楼 405 单元 \\ Phone: +86-21-62489820 \\ Fax: +86-21-62489821
}


(C) 2012 The Author(s). Licensee IntechOpen. This is an open access article distributed under the terms of the Creative Commons Attribution 3.0 License, which permits unrestricted use, distribution, and reproduction in any medium, provided the original work is properly cited. 